\title{
EUV MET Printing and Actinic Imaging Analysis of The Effects of Phase Defects on Wafer CDs
}

Hakseung Han, Stefan Wurm

SEMATECH

Kenneth A. Goldberg, Eric M. Gullikson, Anton Barty* LBNL, LLNL*

Yoshiaki Ikuta, Toshiyuki Uno

AGC

Obert R. Wood II

AMD

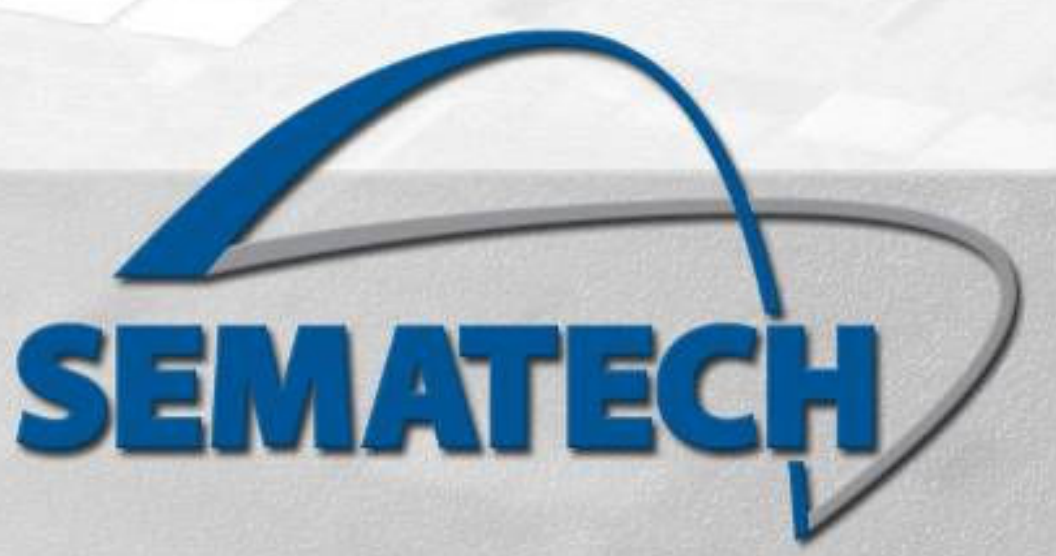

Accelerating the next technology revolution.

Advanced Materials Research Center, AMRC, International SEMATECH Manufacturing Initiative, and ISMI are servicemarks of SEMATECH, Inc. SEMATECH, the SEMATECH logo, Advanced Technology Development Facility, ATDF, and the ATDF logo are registered servicemarks of SEMATECH, Inc. All other servicemarks and trademarks are the property of their respective owners. 


\section{Outline}

- Program defect mask fabrication and structure

- MET exposure condition

- Analysis of substrate pit defect printability

- Focus \& dose effect

- Comparison with simulation

- Programmed pit image from actinic imaging tool 


\section{Printability Study Required for EUVL}

- Mask blank defect spec is a critical issue.

- When to introduce $3^{\text {rd }}$ generation blank inspection tool.

- What size defect can be tolerated?

- SEMATECH has the necessary equipments for this study.

- EUV printing using the EUV microexposure tool (MET)

- Programmed defect mask fabrication and characterization using IBD, inspection, FIB and AFM at the MBDC

- EUV imaging and scanning inspection using SEMATECH-Berkeley actinic inspection tool 


\section{Mask Fabrication and Structure}

- Using Facilities at SEMATECH North MBDC

- FIB milled substrate pits $\rightarrow$ ML deposition $\rightarrow$ FIB milled line

- 9 pit sizes : Depth(a): 2,4,6 nm Width(b): 50, 100, $150 \mathrm{~nm}$

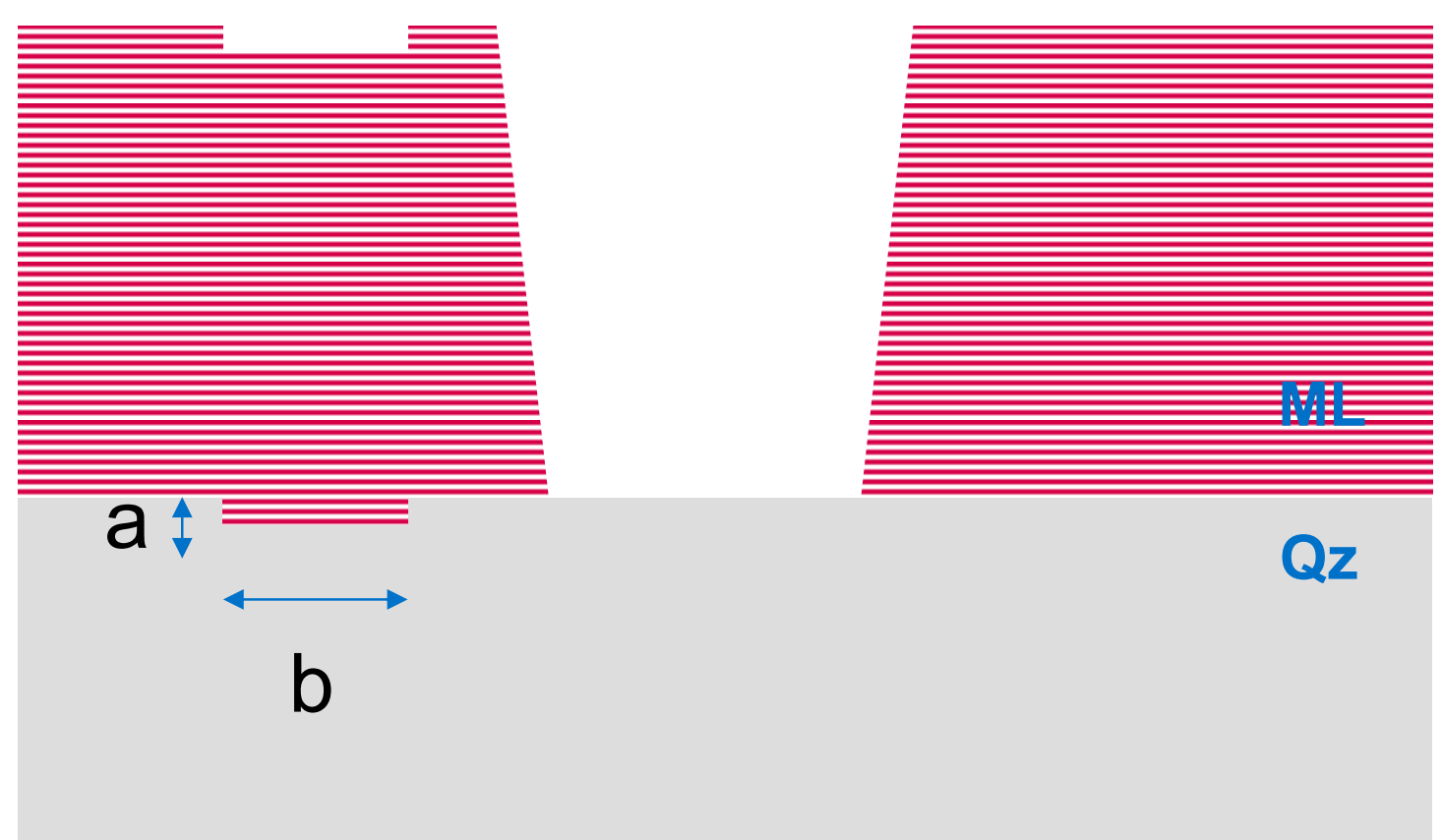




\section{Mask Fabrication and Structure}

- Line image fabricated by FIB-milling of the multilayer

\section{Top View Cross-section View}
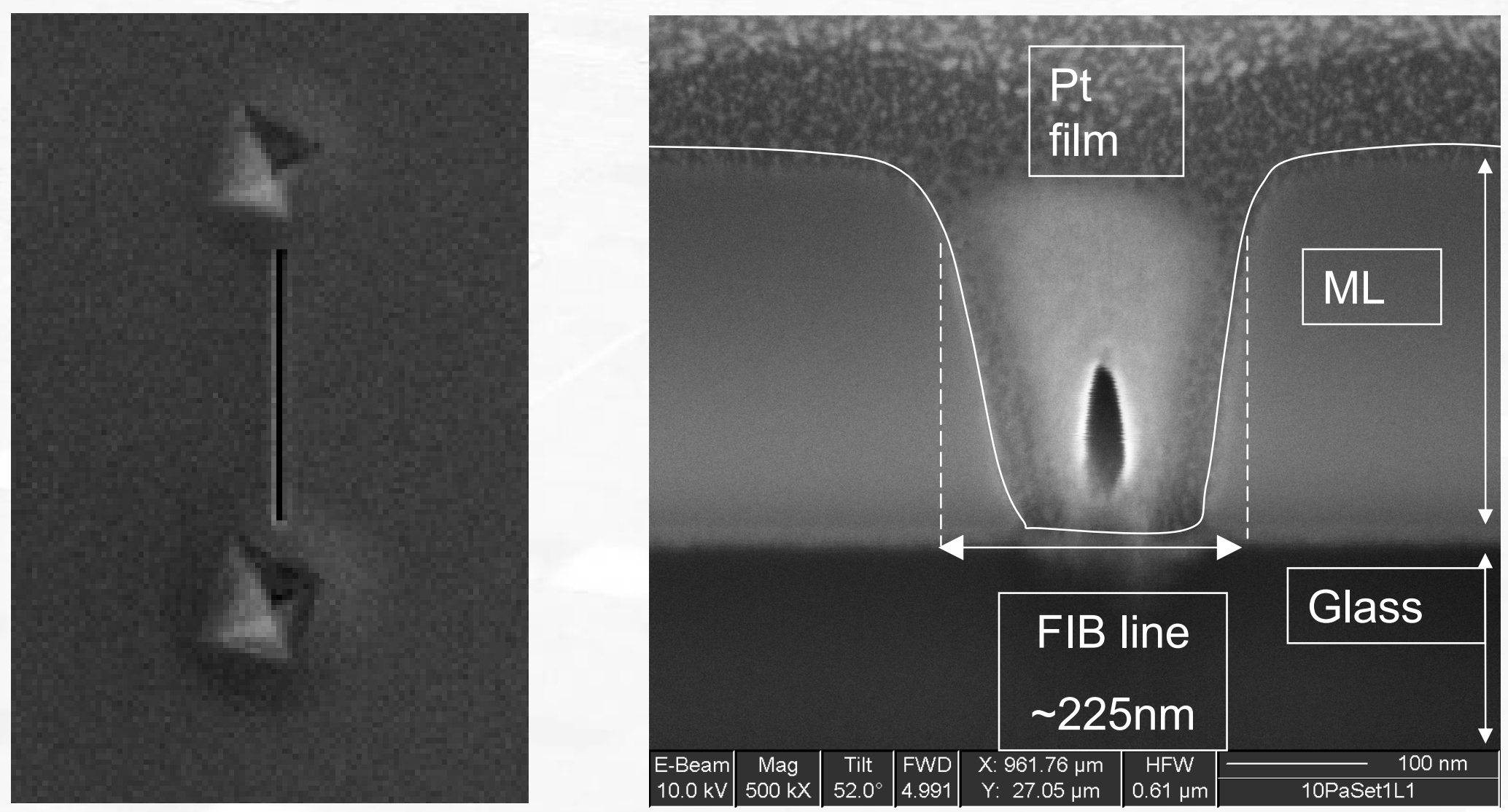


\section{Mask Fabrication and Structure}

- Substrate pits in proximity to isolated line for CD change study

- Lateral pit-to-line spacing varies in $50 \mathrm{~nm}$ steps

- $225 \mathrm{~nm}$ isolated line $-\mathbf{4 5} \mathrm{nm}$ for $5 \mathrm{X}$ exposure

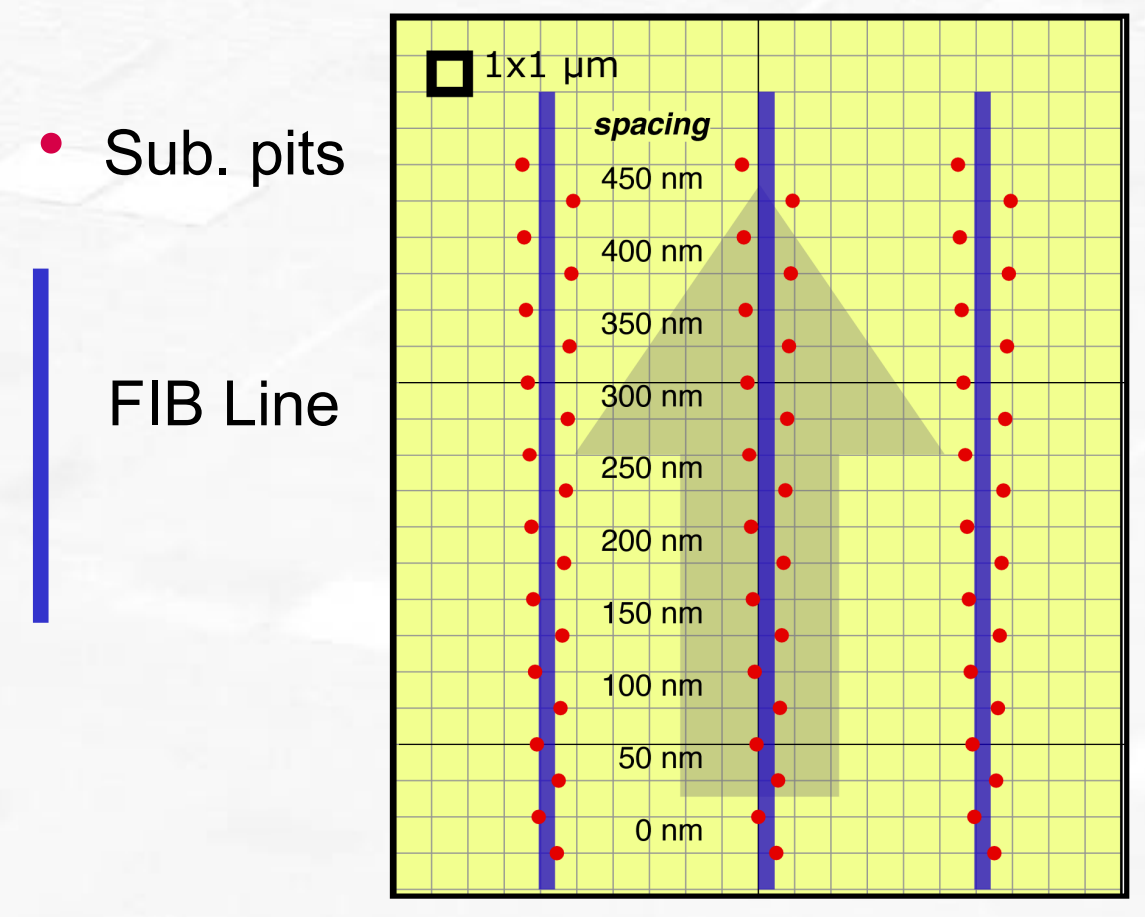

Isolated lines with pits 


\section{Substrate Pit Target size Vs. Measured Size}

- Substrate pit size measurement (AFM)

- Almost the same as the target size

\begin{tabular}{c|c|c|c}
\multicolumn{2}{c|}{ FWHM } & \multicolumn{2}{c}{ Depth } \\
\hline Target & Measured & Target & Measured \\
\hline $50 \mathrm{~nm}(\mathrm{~A})$ & $40-60 \mathrm{~nm}$ & $2 \mathrm{~nm}$ & $2-3 \mathrm{~nm}$ \\
\hline $\begin{array}{c}100 \mathrm{~nm} \\
(\mathrm{~B})\end{array}$ & $75-90 \mathrm{~nm}$ & $4 \mathrm{~nm}$ & $4-5 \mathrm{~nm}$ \\
\hline $\begin{array}{c}150 \mathrm{~nm} \\
(\mathrm{C})\end{array}$ & $\begin{array}{c}150-170 \\
\mathrm{~nm}\end{array}$ & $6 \mathrm{~nm}$ & $6-9 \mathrm{~nm}$ \\
& & &
\end{tabular}

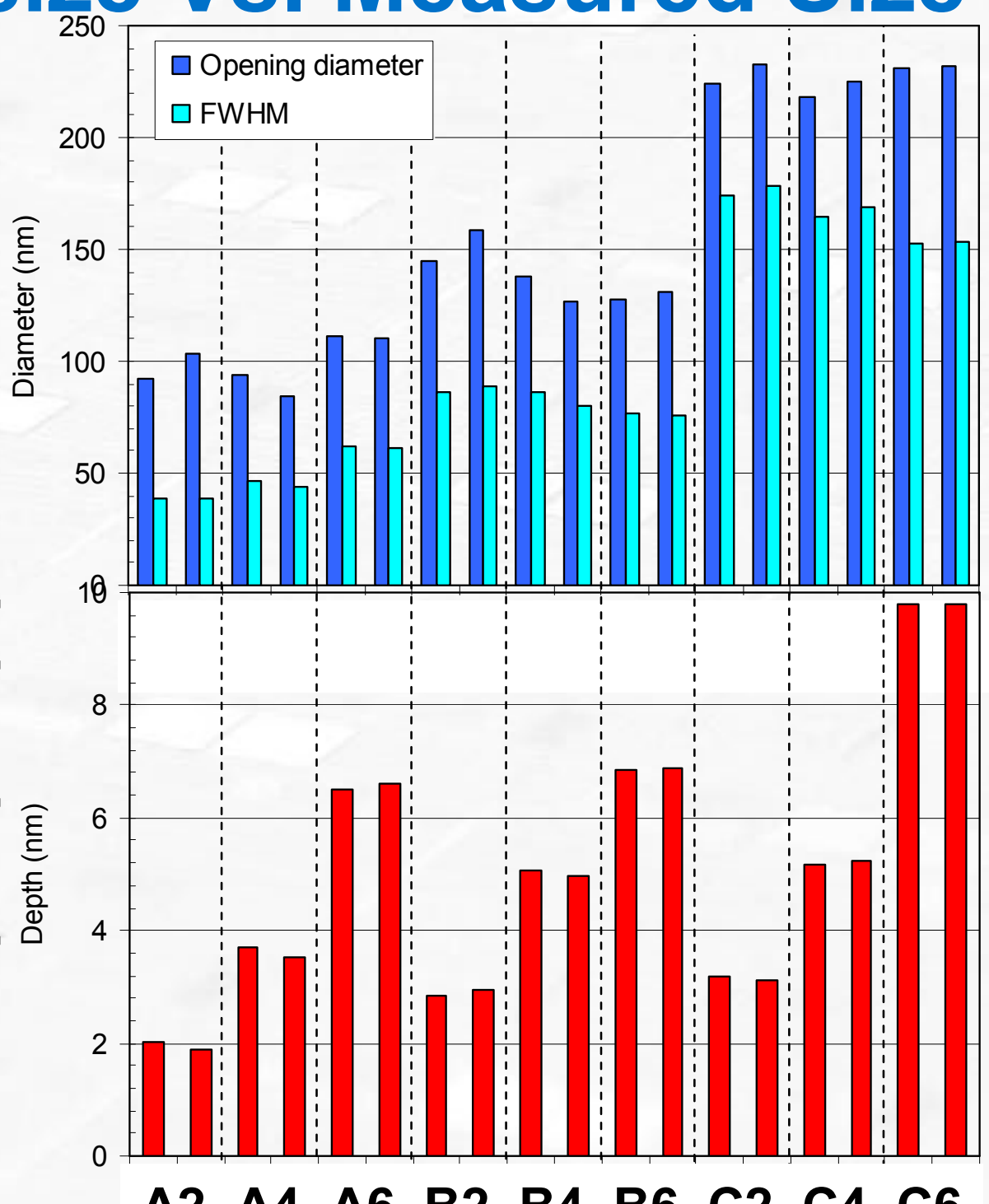

A2 A4 A6 B2 B4 B6 C2 C4 C6

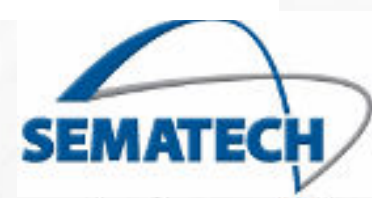




\section{Substrate Pit Size Change After ML Deposition}

- Pit size before/after deposition - AFM scanned images

- Deposition by MBDC IBD tool: Wider FWHM and lower depth after depo. But the change is less than $20 \%$.

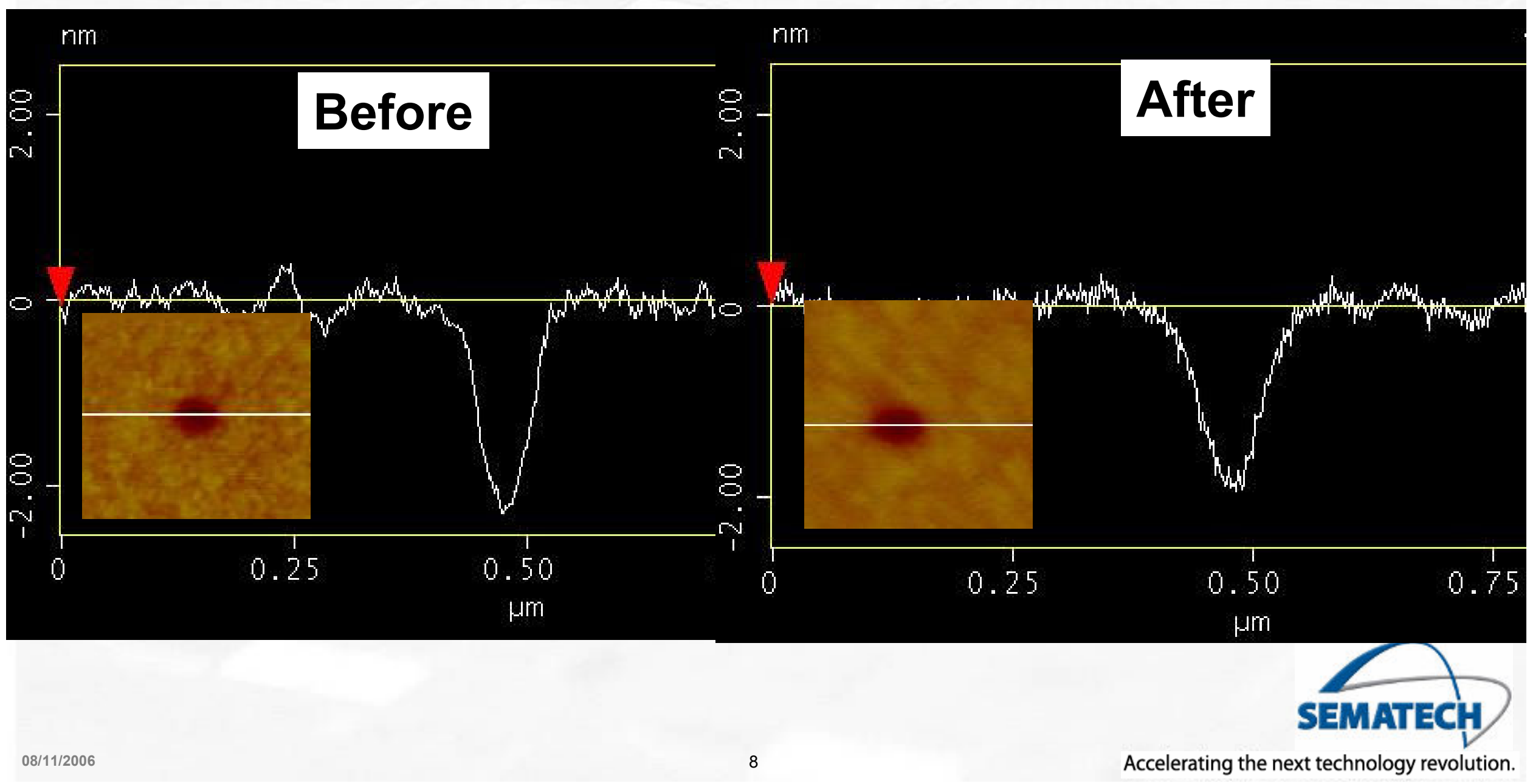




\section{MET Exposure Condition}

- Exposure

- SEMATECH Berkeley MET

- 5X demagnification, 0.3NA

- Rotated Dipole, R\&H resist

- Dose focus split $19 \times 11$

- Dose 10.6 / 5\%

- Focus 4675 / 50 nm

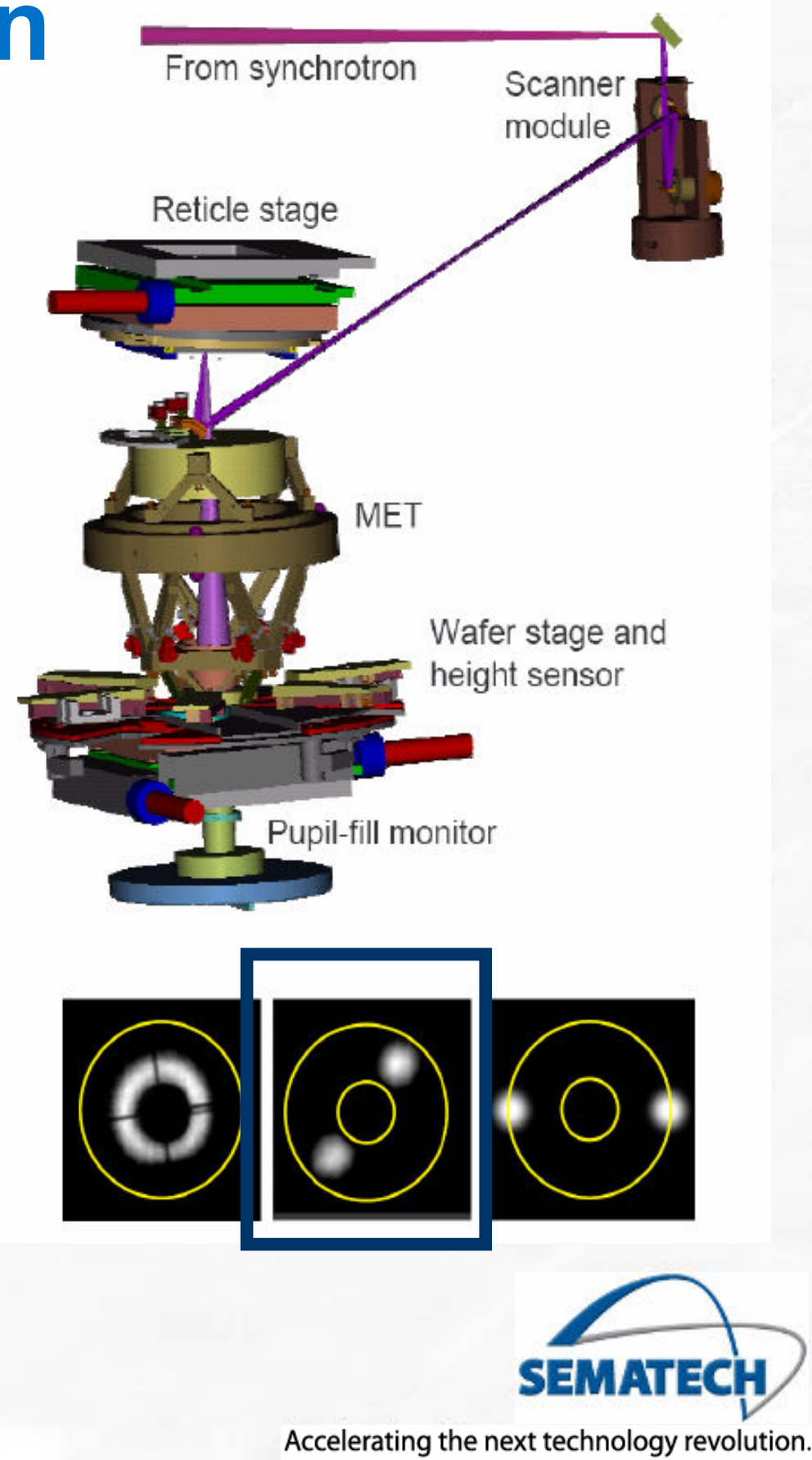




\section{Printability of Substrate Pits}

- Substrate pits $150 \mathrm{~nm}$ wide and $6 \mathrm{~nm}$ deep were clearly printed

- 6 pits printed on one side $\rightarrow$ Pits $200 \mathrm{~nm}$ from the line are printable

- All $150 \mathrm{~nm}$ wide pits were printed down to $2 \mathrm{~nm}$ deep

\section{$150 \mathrm{~nm}$ W $6 \mathrm{~nm} D$}

$400 \mathrm{~nm}$

wafer

dimension 


\section{Printability of Substrate Pits}

- Substrate pits $100 \mathrm{~nm}$ wide and $6 \mathrm{~nm}$ deep

- Only 3 pits were printed. This means pits up to about $80 \mathrm{~nm}$ from the line are printable.

$100 \mathrm{~nm} \mathrm{~W} 6 \mathrm{~nm} \mathrm{D}$

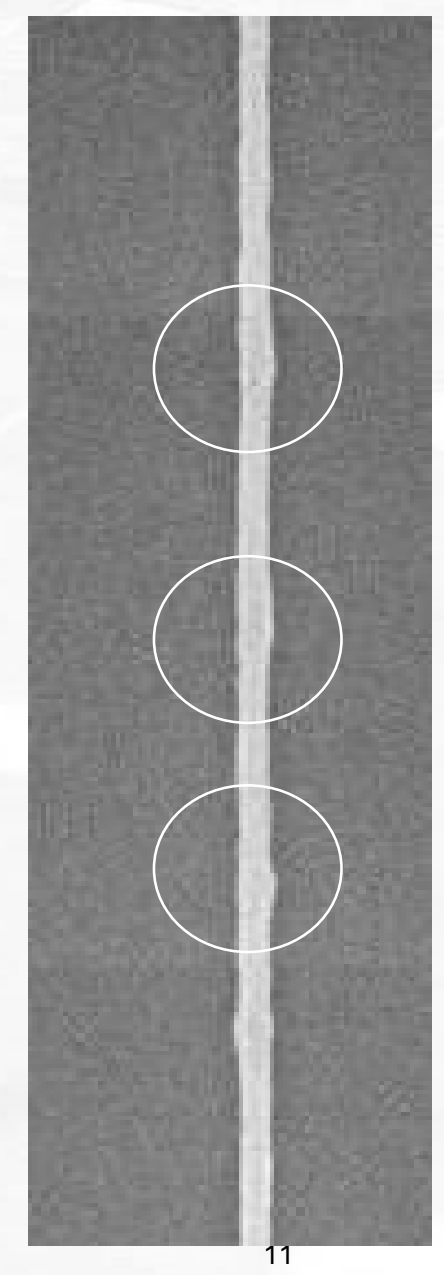




\section{Printability of Substrate Pits}

- Substrate pits $100 \mathrm{~nm}$ wide and $2 \mathrm{~nm}$ deep

- Not printable but printability depends on focus and dose

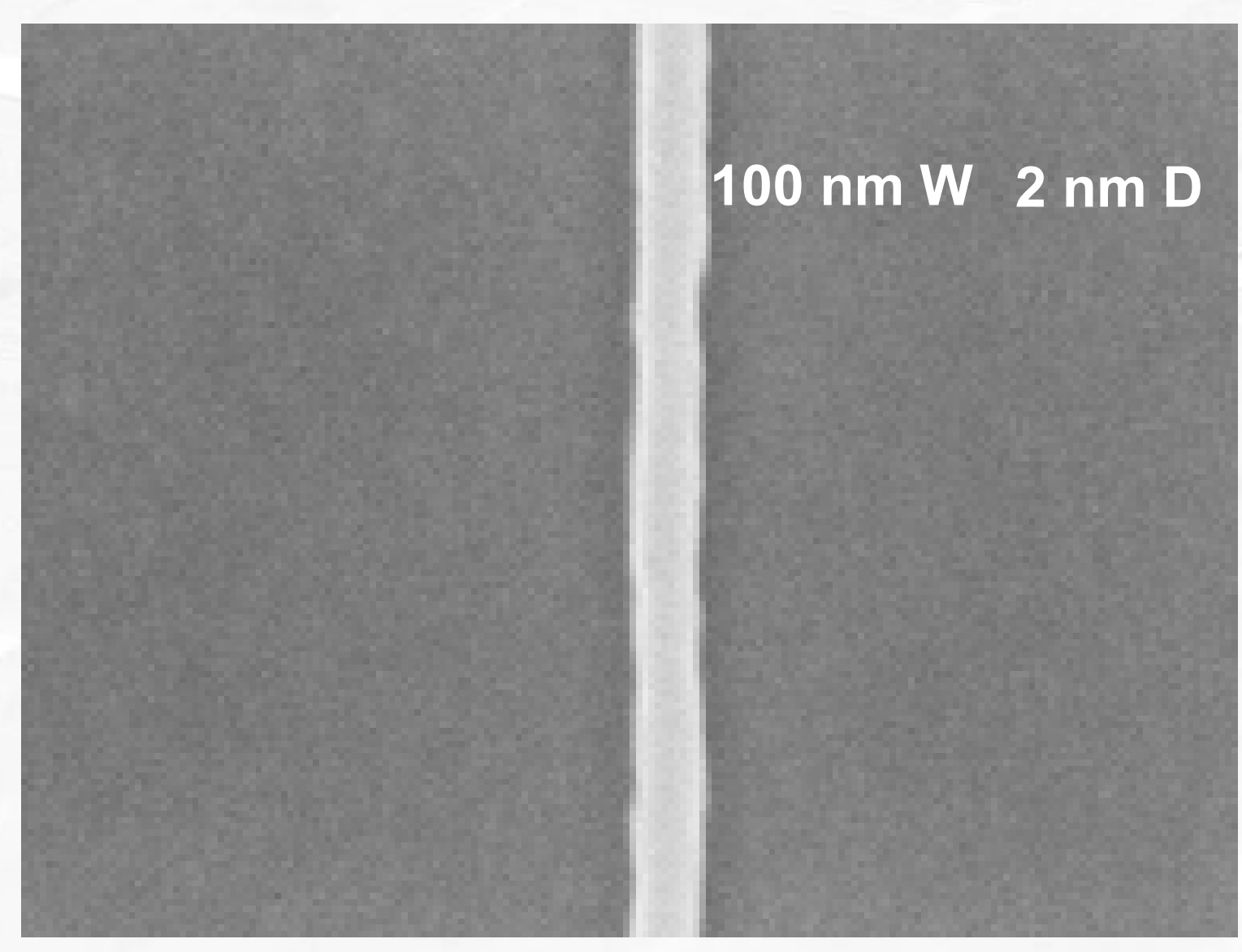

\section{Focus \& Dose effect}




\section{Through Focus}

- Defocus makes pits a little more printable

$150 \mathrm{~nm} \mathrm{~W} 6 \mathrm{~nm} D$

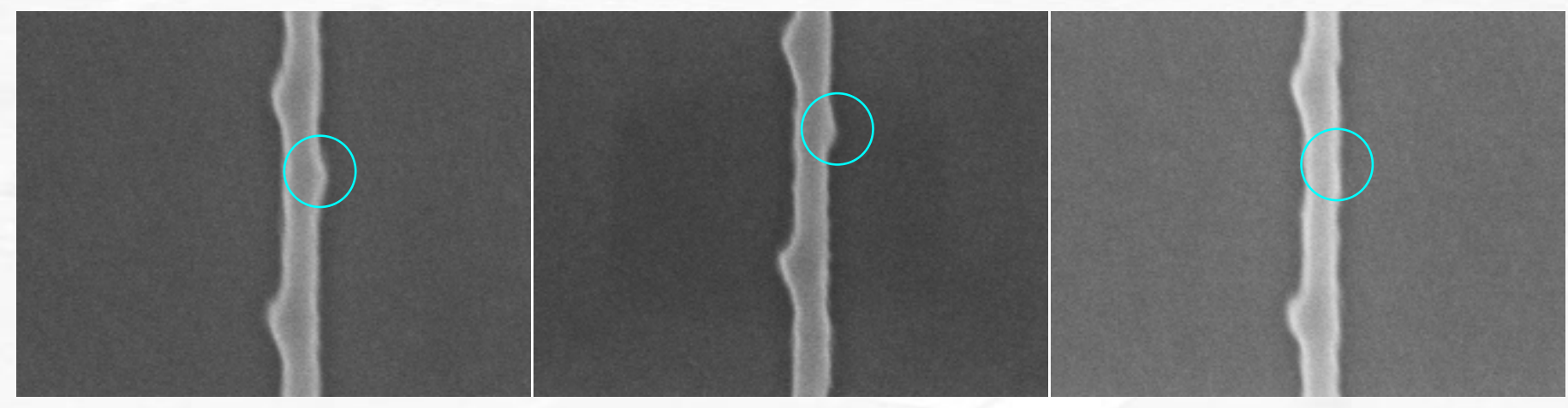

$-250 \mathrm{~nm}$

$-200 \mathrm{~nm}$

$-150 \mathrm{~nm}$

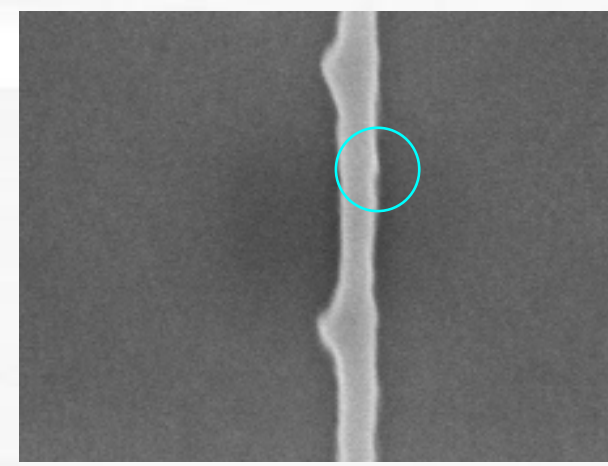

$-100 \mathrm{~nm}$

$-50 \mathrm{~nm}$
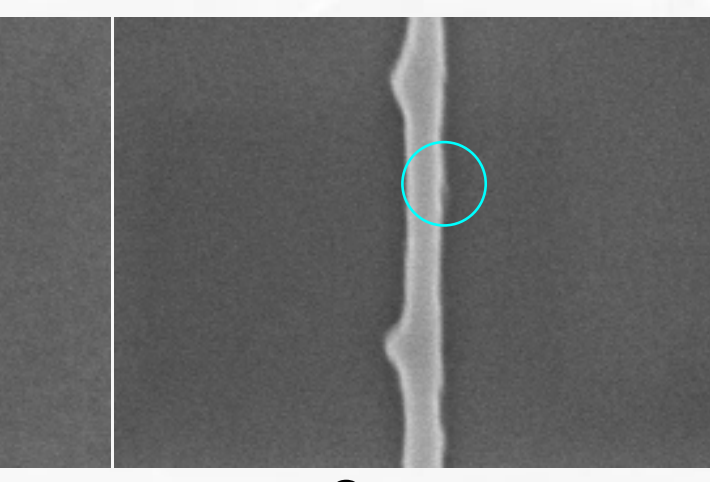

$0 \mathrm{~nm}$ 


\section{Through Dose}

- Low dose makes pits more printable

150 nm W 6 nm D
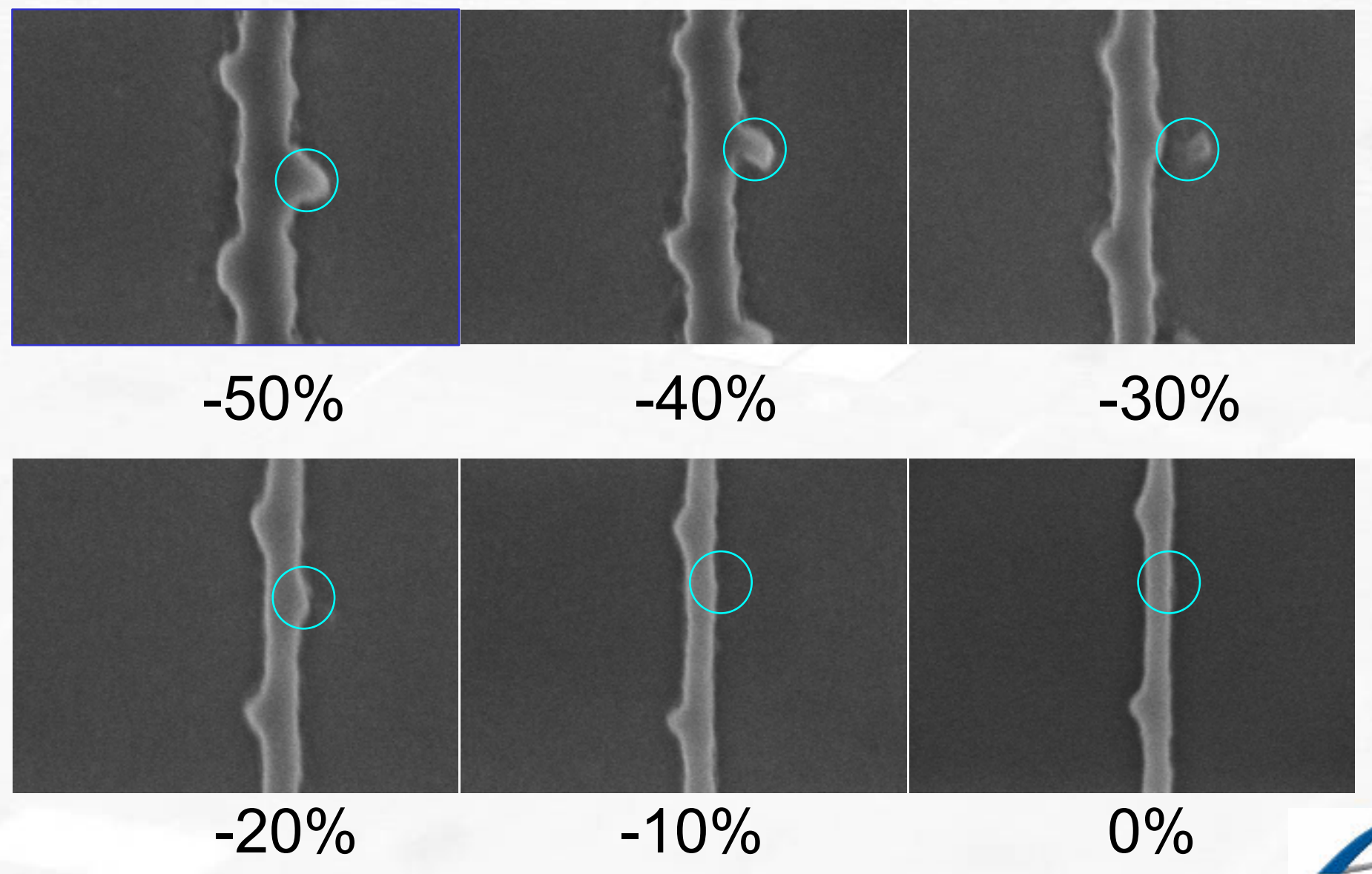


\section{Printability According to Dose and Focus}

- Defocus images (-100nm)

- $100 \mathrm{~nm} \times 2 \mathrm{~nm}$ pits look printable only at $-5 \%$ dose

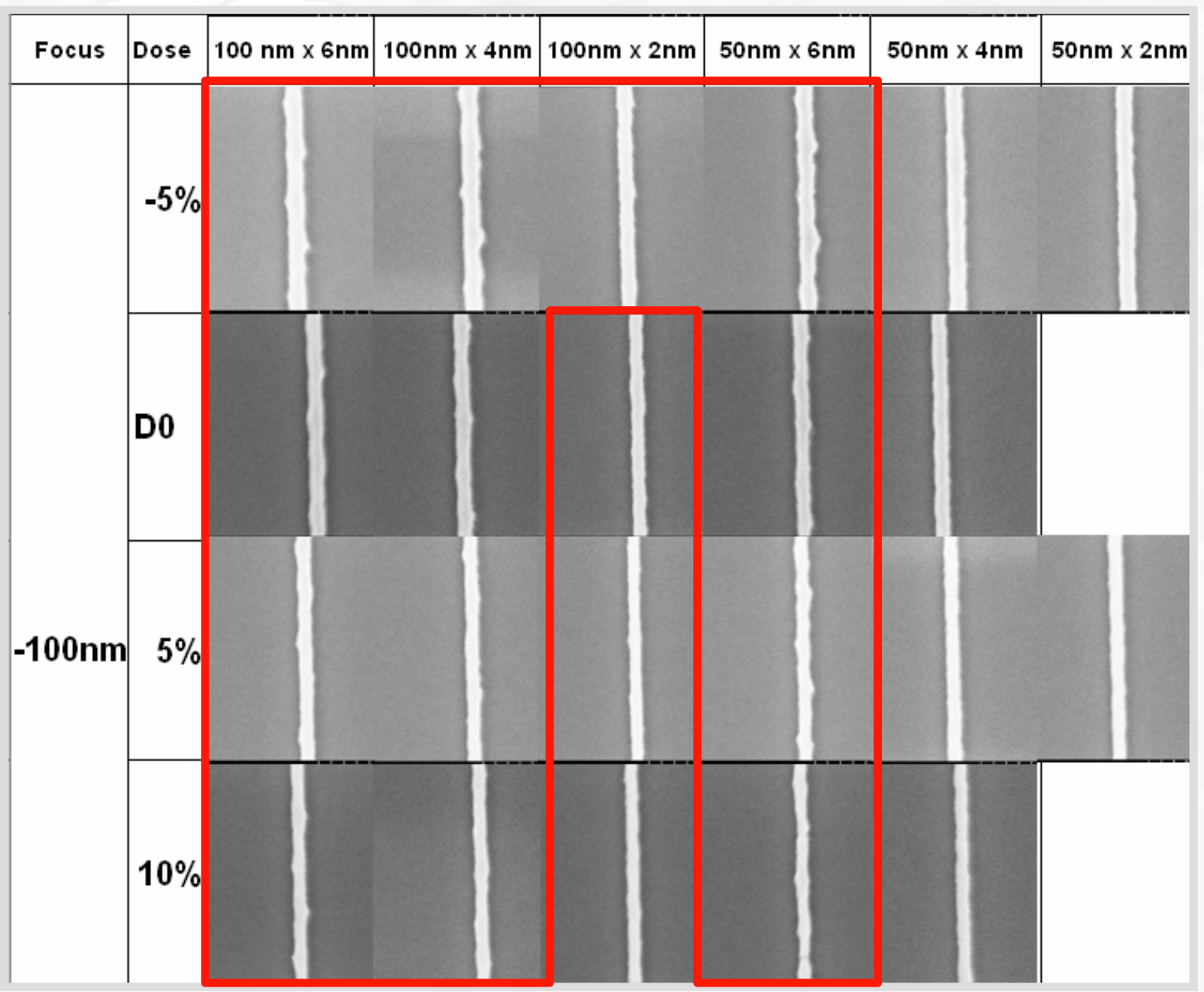

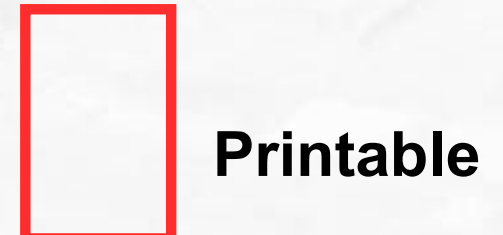

Assumption : two defects with $400 \mathrm{~nm}$ spacing regardless of $C D$ change magnitude

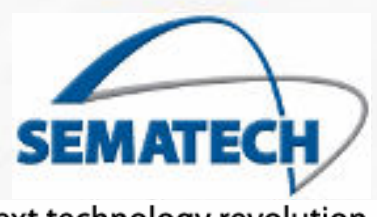




\section{Printability According to Dose and Focus}

- At just focus, some pits are not printable

- Only 2 pits are printable, pits $40 \mathrm{~nm}$ from the line are printable.

- Printability depend more on depth than width

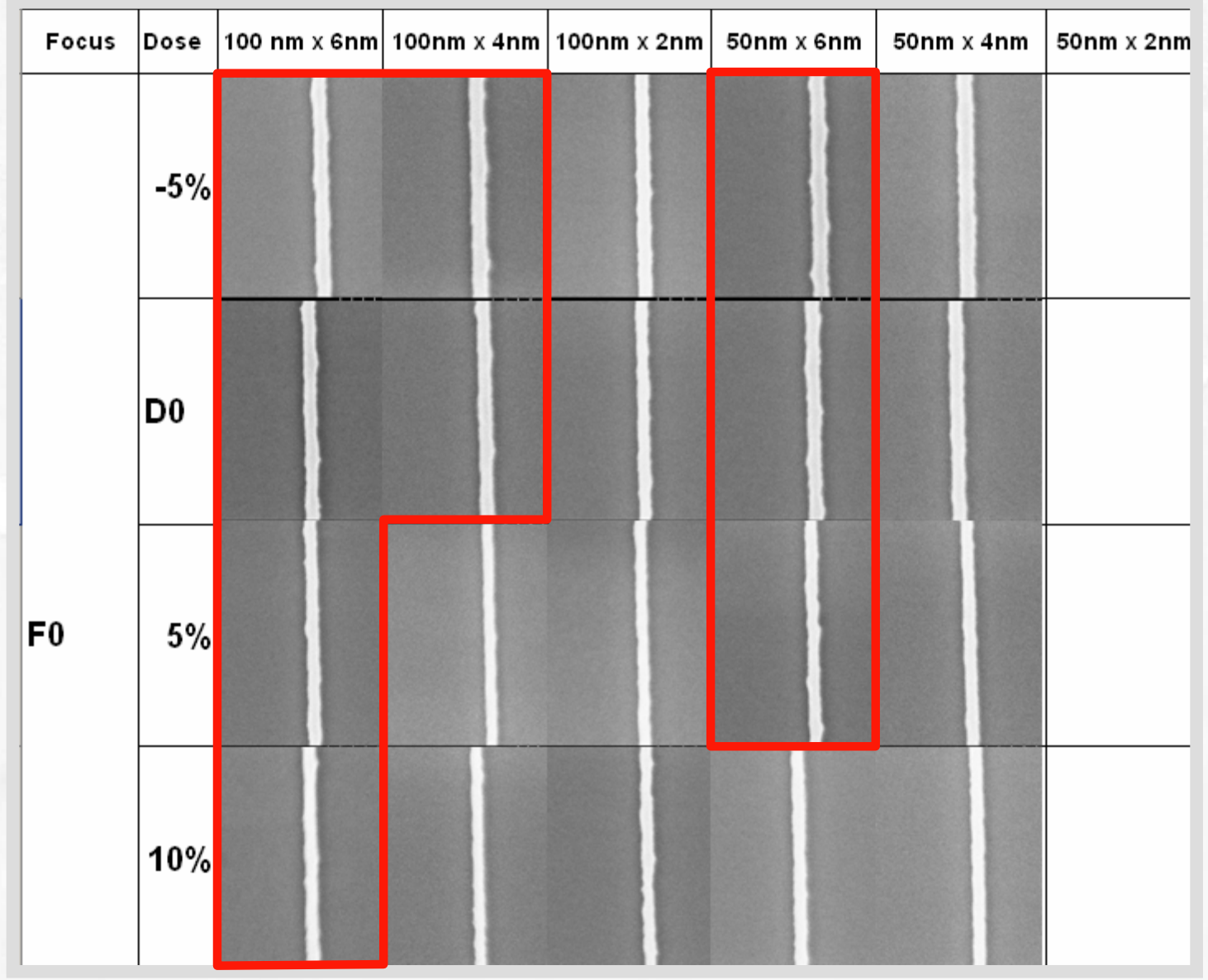




\section{Defect Printability - Simulation}

- Aerial image simulation as a function of defect FWHM, height and focus

- Clearly showing printability change according to focus

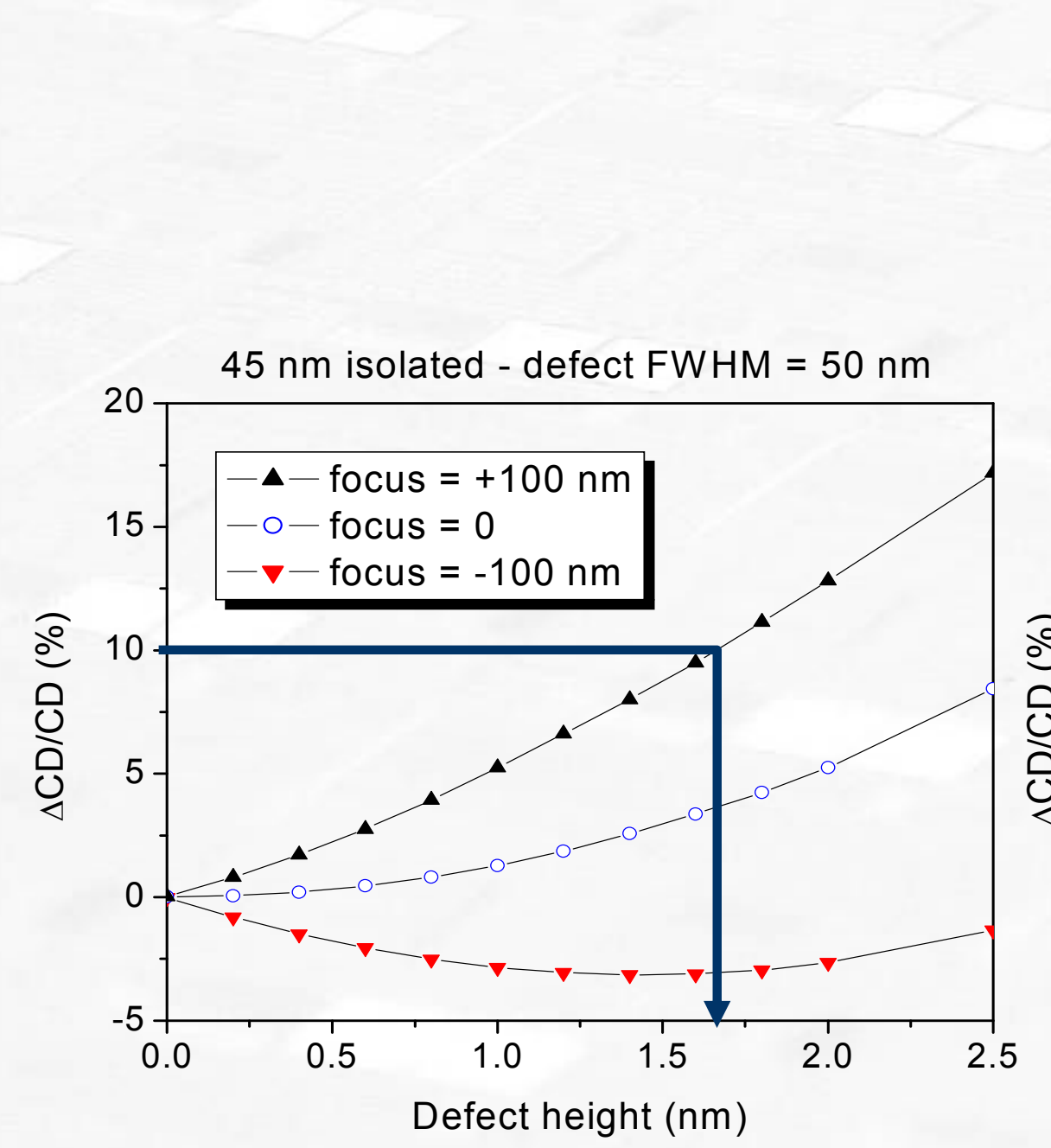

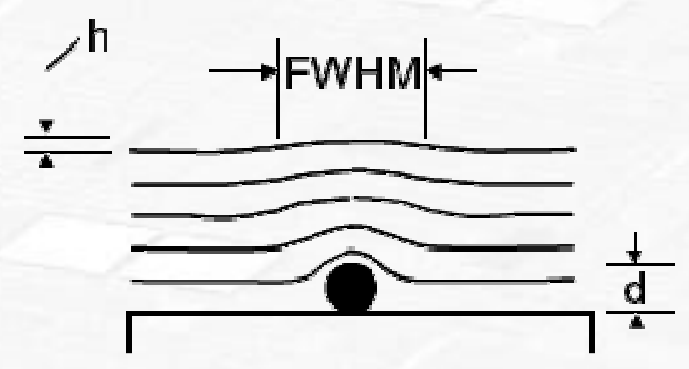

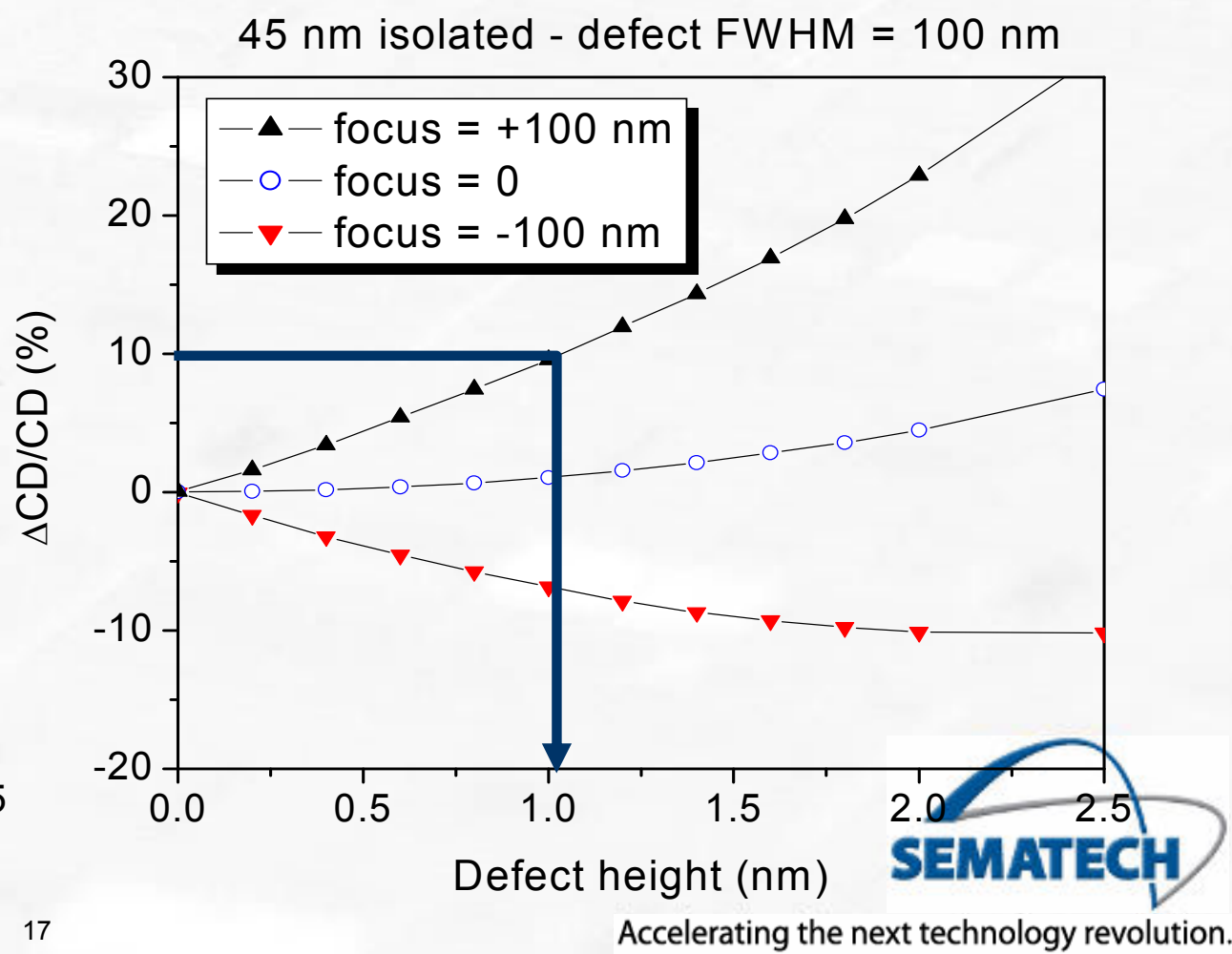




\section{Comparison of Simulation and Real Test}

- Comparison at $100 \mathrm{~nm}$ defocus

- Well matched at large defect sizes, 1-2 $\mathrm{nm}$ difference at smaller defects

- Bake diffusion, develop process OR exposure condition difference btw simulation and test

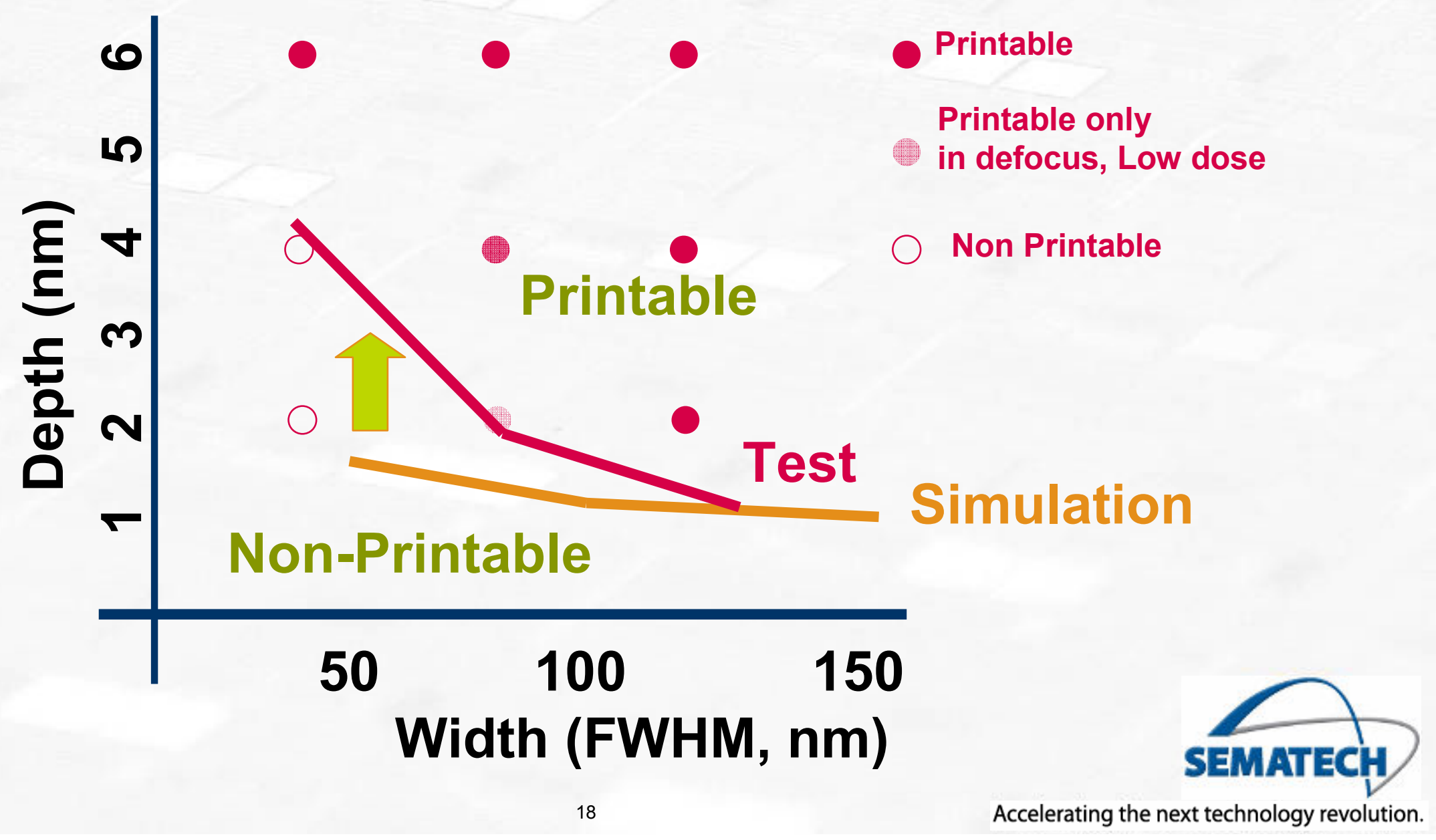




\section{Comparison of Simulation and Real Test}

- Comparison at $100 \mathrm{~nm}$ defocus

- Well matched at large defect sizes, 1-2 $\mathrm{nm}$ difference at smaller defects

- Bake diffusion, develop process OR exposure condition difference btw simulation and test

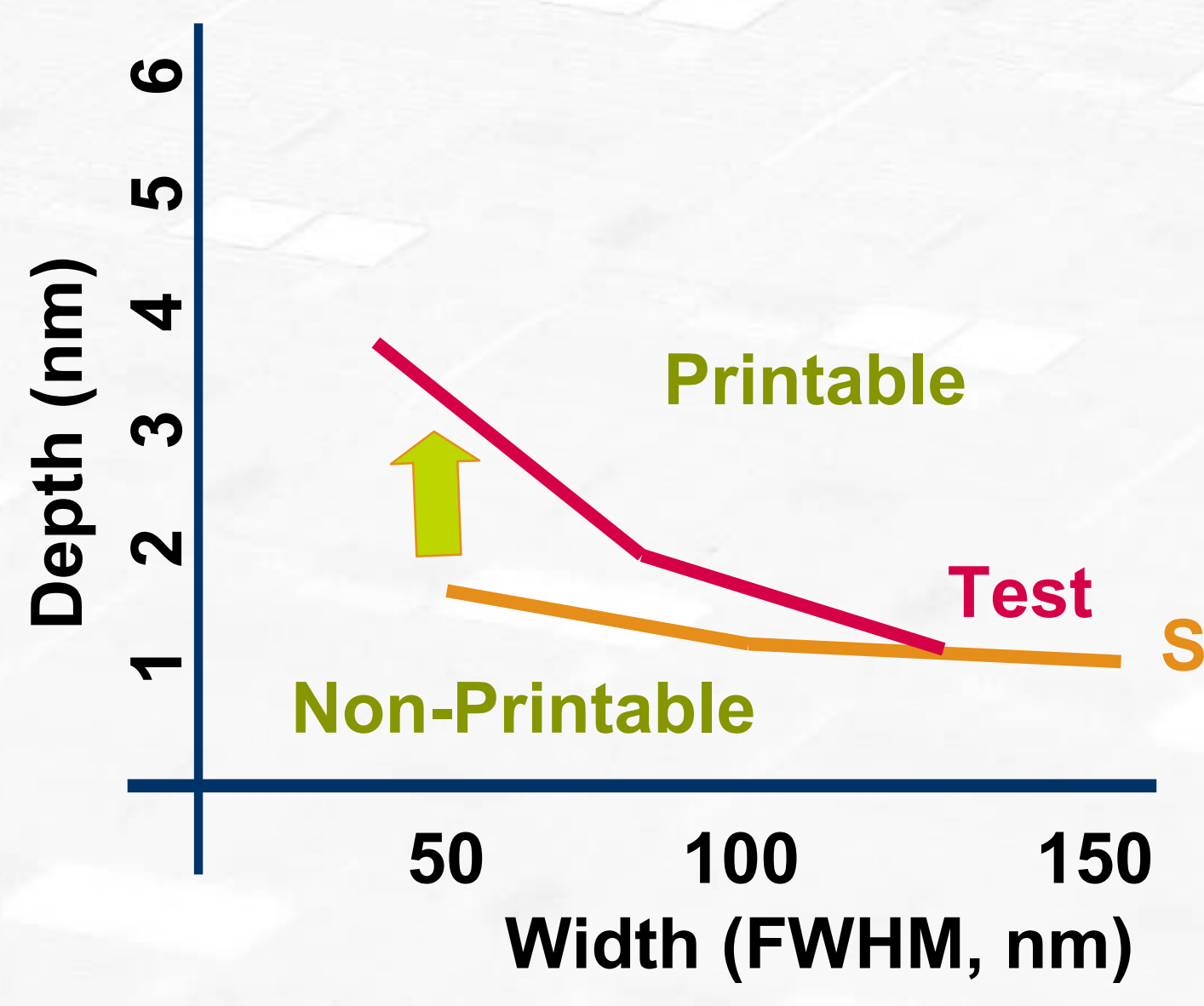




\section{Comparison with EUV Imaging}

- EUV image from SEMATECH-Berkeley actinic tool

- Clearly shows substrate pits

- Upgrading to get reliable CD measurement

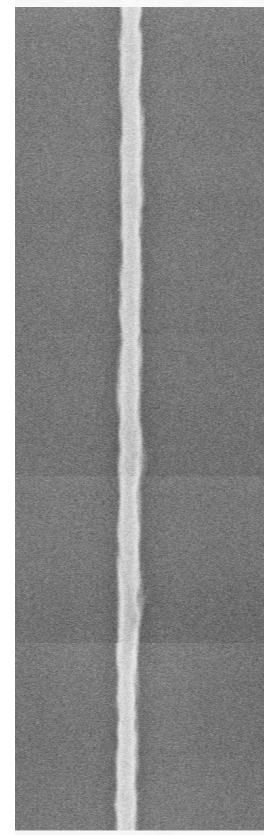

Resist SEM image On a wafer

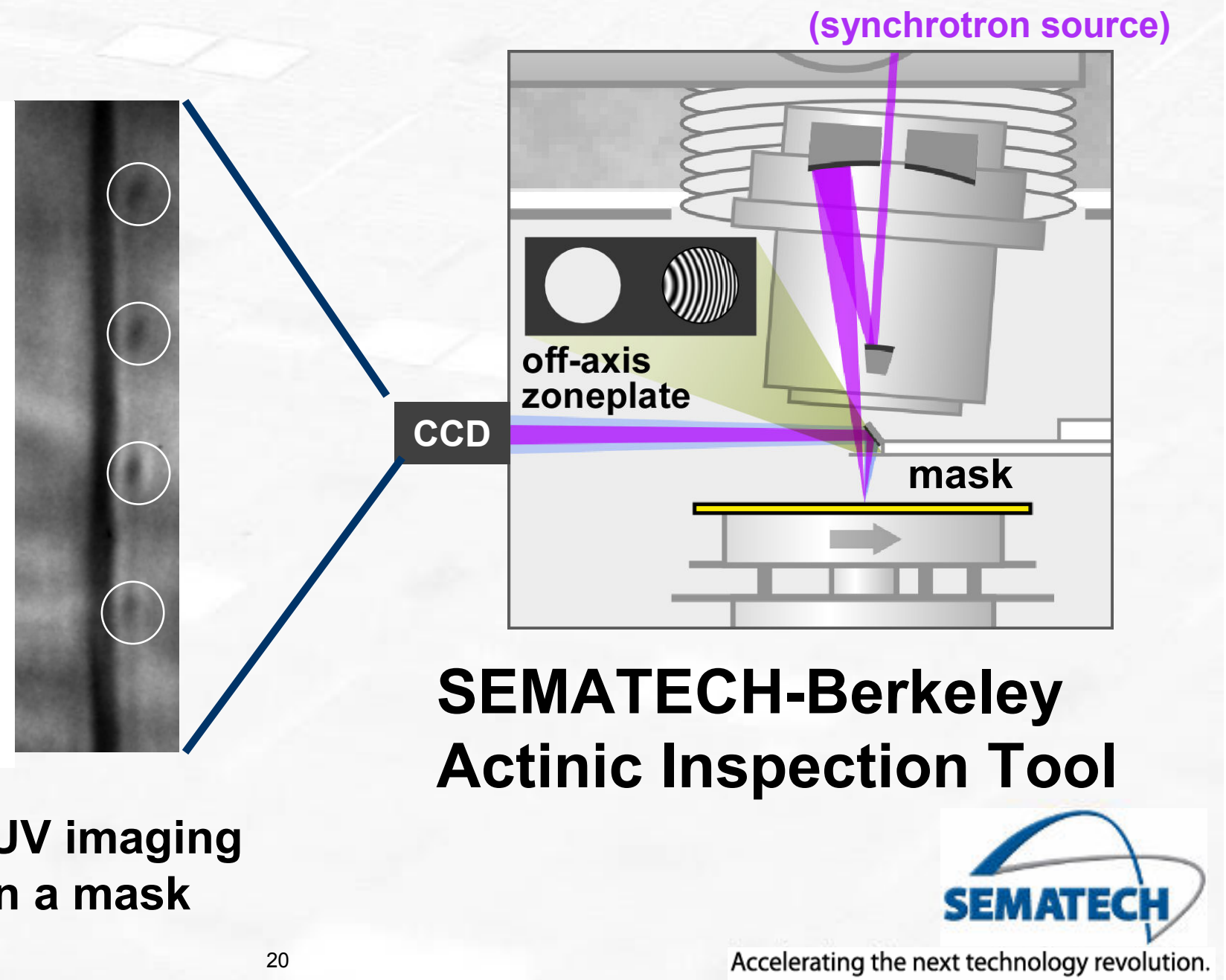




\section{Summary}

- Printability study using SEMATECH EUV infrastructure

- Below $100 \mathrm{~nm} \times 6 \mathrm{~nm}$ pit size, printable pit-to-line edge spacing is only $40 \mathrm{~nm}$

- $50 \mathrm{~nm}$ wide substrate pits up to $4 \mathrm{~nm}$ deep were not printable

- More optimistic than aerial image simulation

- Planning comparison study of printing image and EUV image with New program defect mask and upgraded actinic inspection tool.

- Ultimately determining exact $32 \mathrm{~nm}$ HP printable blank defect size and spec. 


\section{Acknowledgement}

- SEMATECH MBDC deposition, integration and cleaning team.

- Berkeley EUV MET team.

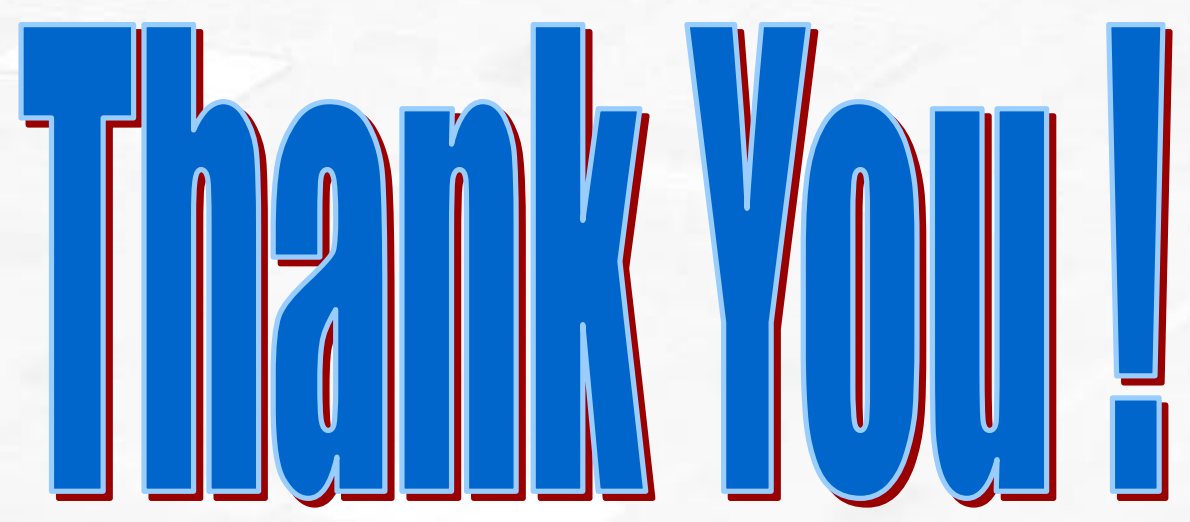

This work was performed under the auspices of the U. S. Department of Energy by University of California, Lawrence Livermore national Laboratory under Contract W-7405-Eng-48. 